\title{
Predicting Long-Term Global Outcome after Traumatic Brain Injury: Development of a Practical Prognostic Tool Using the Traumatic Brain Injury Model Systems National Database
}

\author{
William C. Walker, ${ }^{1}$ Katharine A. Stromberg, Jennifer H. Marwitz, Adam P. Sima, \\ Amma A. Agyemang, Kristin M. Graham, ${ }^{1}$ Cynthia Harrison-Felix, Jeanne M. Hoffman, \\ Allen W. Brown, Jeffrey S. Kreutzer, and Randall Merchant ${ }^{1}$
}

\begin{abstract}
For patients surviving serious traumatic brain injury (TBI), families and other stakeholders often desire information on longterm functional prognosis, but accurate and easy-to-use clinical tools are lacking. We aimed to build utilitarian decision trees from commonly collected clinical variables to predict Glasgow Outcome Scale (GOS) functional levels at 1, 2, and 5 years after moderate-to-severe closed TBI. Flexible classification tree statistical modeling was used on prospectively collected data from the TBI-Model Systems (TBIMS) inception cohort study. Enrollments occurred at 17 designated, or previously designated, TBIMS inpatient rehabilitation facilities. Analysis included all participants with nonpenetrating TBI injured between January 1997 and January 2017. Sample sizes were 10,125 (year-1), 8,821 (year-2), and 6,165 (year-5) after cross-sectional exclusions (death, vegetative state, insufficient post-injury time, and unavailable outcome). In our final models, post-traumatic amnesia (PTA) duration consistently dominated branching hierarchy and was the lone injury characteristic significantly contributing to GOS predictability. Lower-order variables that added predictability were age, pre-morbid education, productivity, and occupational category. Generally, patient outcomes improved with shorter PTA, younger age, greater pre-morbid productivity, and higher pre-morbid vocational or educational achievement. Across all prognostic groups, the best and worst good recovery rates were $65.7 \%$ and $10.9 \%$, respectively, and the best and worst severe disability rates were $3.9 \%$ and $64.1 \%$. Predictability in test data sets ranged from C-statistic of 0.691 (year-1; confidence interval [CI], 0.675, 0.711) to 0.731 (year-2; CI, 0.724, 0.738). In conclusion, we developed a clinically useful tool to provide prognostic information on long-term functional outcomes for adult survivors of moderate and severe closed TBI. Predictive accuracy for GOS level was demonstrated in an independent test sample. Length of PTA, a clinical marker of injury severity, was by far the most critical outcome determinant.
\end{abstract}

Keywords: classification tree; functional outcome; Glasgow Outcome Scale; prognosis; traumatic brain injury classification tree

\section{Introduction}

$\mathbf{T}$ Traumatic brain inJury (TBI) is a complex condition with a broad spectrum of injury severity and locations, and highly variable outcomes. For survivors of moderate-severe TBI, recovery is often protracted, and functional outcomes over time can range from total dependence to full recovery. This heterogeneity has undoubtedly contributed to disappointing results in intervention trials. ${ }^{1,2}$ Whereas this prognostic uncertainty may offer hope for more severely injured individuals, it also provokes anxiety about the future and hampers setting realistic expectations and plans. ${ }^{3}$ Unfortunately, TBI outcomes research has not well informed the accurate prediction of long-term, meaningful, functional outcomes. ${ }^{4}$ For TBI severities greater than mild, the standard clinical prognosis has remained "time will tell" or "we honestly don't know."

Limitations of existing prediction models for TBI are quite profound, even for the most widely used global outcome measures, Glasgow Outcome Scale (GOS) ${ }^{5}$ and extended GOS (GOSE). ${ }^{6}$

Departments of ${ }^{1}$ Physical Medicine and Rehabilitation and ${ }^{2}$ Biostatistics, Virginia Commonwealth University, Richmond, Virginia.

${ }^{3}$ Traumatic Brain Injury Model Systems National Data and Statistical Center, Craig Hospital, Englewood, Colorado.

${ }^{4}$ Department of Rehabilitation Medicine, University of Washington, Seattle, Washington.

${ }^{5}$ Department of Physical Medicine and Rehabilitation, Mayo Clinic, Rochester, Minnesota.

(c) William C. Walker et al., 2018; Published by Mary Ann Liebert, Inc. This Open Access article is distributed under the terms of the Creative Commons Attribution Noncommercial License (http://creativecommons.org/licenses/by-nc/4.0/) which permits any noncommercial use, distribution, and reproduction in any medium, provided the original author(s) and the source are credited. 
Published models are plagued by deficiencies, ${ }^{7,8}$ including development from small sample sizes and limited discriminative ability and calibration when applied to independent data. ${ }^{8}$ Some models are based on complex mathematical formulas that prohibit efficient clinical use and/or make assumptions that prohibit flexible modeling. Most are limited to short-term prognostication to 1 year postinjury or far less, and fail to address long-term functional outcomes.

Classification tree methodology is especially suited for building a utilitarian prediction model because it relies on simple branching logic rather than complex mathematical formulas that impede clinical translation. However, a literature review found that classification trees are rarely used in TBI prognostic modeling. ${ }^{9,10}$ The single publication arising from the TBI Model Systems (TBIMS), the epicenter for studying moderate-to-severe TBI functional outcomes, used recursive portioning for multiple outcomes and has several important limitations: 1) Outcomes beyond 1 year were not studied; 2) sample sizes were limited (year-1 models ranged from 1361 to 1817); and 3) modeling was constrained to two-way splitting. ${ }^{11}$ In fact, all published prognostic models for TBI outcomes using classification tree algorithms similarly assume binary splits by continuous and categorical covariates, meaning each branching node (i.e., a group of homogenous participants) delivers only two branches. ${ }^{7}$ This exclusive binary split assumption does not maximize the data use and may result in an important loss of predictability. ${ }^{8}$

Using the TBIMS National Database (NDB), this study sought to address this research gap by developing a clinically useful prognostic tool for meaningful long-term functional outcomes through an innovative strategy that permits more than two-way node splitting based on a data-driven decision rule rather than an analyst-induced decision rule. Our specific aims were to: 1) build decision tree models for functional GOS levels at post-injury year 1,2 , and 5 in persons with moderate-to-severe closed TBI and 2) test their predictability within independent data sets.

\section{Methods}

\section{Participants}

Participants were all consented and enrolled in the National Institute on Disability, Independent Living, and Rehabilitation Research (NIDILRR) funded TBIMS NDB, a collaborative, multicenter clinical research effort in the United States initiated in $1987 .{ }^{12}$ All 16 currently enrolling plus one formerly enrolling centers had institutional review board approval. TBIMS eligibility criteria were: 1) sustained TBI with at least one of the following characteristics: Glasgow Coma Scale (GCS) score $<13$ on emergency admission (not attributed to intubation, sedation, or intoxication), loss of consciousness $>30$ min (not attributed to sedation or intoxication), post-traumatic amnesia (PTA) $>24 \mathrm{~h}$, or trauma-related intracranial abnormality on neuroimaging; 2) 16 years or older; 3 ) presented to a designated TBIMS acute care hospital within $72 \mathrm{~h}$ of injury; and 4) received acute rehabilitation care within the TBIMS.

Exclusion criteria for our analyses included injury dates before January 1997 because outcome predictors differ in the earlier years of the TBIMS, ${ }^{13}$ and penetrating TBI as previously operationally defined, ${ }^{14}$ because of its rarity and unique outcome patterns. ${ }^{14}$ Individuals were excluded from a given analysis year, and future years, if they had not reached that time point post-injury or if they had withdrawn. Those whose GOS score was otherwise unavailable at an outcome time point were removed from that year's analysis. Given the rehabilitation setting for enrollment, focus on functional outcomes, and rarity of these outcomes, individuals were also excluded from a given analysis year if they had died beforehand and were removed from that year's analysis if vegetative. To maximize sample sizes and minimize sampling bias, individuals may have been included in one cross-sectional analysis but not in other year(s). Figure 1 displays the participant flow into the final analysis data sets. Exclusions are labeled as "excluded" versus "removed" depending on viability of collecting future GOS. The excluded categories were death, withdrew consent, or insufficient time elapsed post-injury (i.e., premature). The removed categories were vegetative state, refused, incarcerated, enrollment site lost funding, lost (unable to contact), missing predictor (test data set only), or incomplete (assessment conducted but missing GOS). Almost all incomplete classifications were from the optional use of the mail-in version of the TBIMS follow-up assessment, which lacks the GOS interview. The final data sets for the 1-, 2-, and 5-year GOS had 10,125, 8821, and 6165 participants, respectively.

\section{Outcomes}

The GOS, ${ }^{15}$ a 5 -point ordinal scale, is long considered the gold-standard measure of global outcome after TBI. ${ }^{5}$ It has broad applicability and clear, concise, and functionally meaningful categories. ${ }^{15,16}$ The GOSE is administered in the TBIMS NDB and was designed to increase responsiveness of the GOS for research applications and bifurcates the three functional GOS categories, severe disability (SD), moderate disability (MD), and good recovery (GR). We collapsed the GOSE into the original GOS to enhance comparisons with past research and provide distinct and more understandable categorizations for clinicians, patients, and families (see Table 1 for descriptors).

\section{Predictors}

To avoid the potential for an overly cumbersome tool, we parsimoniously selected independent variables that had demonstrated predictive value in past research and were readily accessible to clinicians. Patient demographic variables included age at injury, sex, and education level (less than high school, high school/GED [General Educational Development], or more than high school). ${ }^{17,18}$ Race was not included because findings on its additive predictive value beyond other socioeconomic factors have been mixed, ${ }^{19,20}$ and because the increasing diversity of racial identification would impede clinical classification of many persons.

Pre-injury health and socioeconomic characteristics included were past TBI from the OSU TBI-ID interview (binary present/ absent and number of TBIs), ${ }^{21,22}$ productivity (yes/no with yes identified as competitive employment, full-time student, or running a household), occupational category (professional, skilled, manual, or unemployed), ${ }^{23}$ and problem alcohol and illicit drug use history (both yes/no). Problem alcohol use was measured on the Behavioral Risk Factors Surveillance System Questionnaire from October 1999 to present, ${ }^{24}$ and preceding this date was extrapolated from the Quantity-Frequency-Variability Index. ${ }^{25}$

Clinical measures of brain injury severity included were initial Glasgow Coma Scale (GCS) motor score ${ }^{26}$ and post-traumatic amnesia (PTA) duration, ${ }^{13}$ measured continuously. A binary PTA variable also indicated status at discharge from inpatient rehabilitation (yes/no still in PTA), with total length of stay (LOS) from injury through inpatient rehabilitation serving as the continuous measure of PTA if yes; this permitted maximum inclusiveness and avoided nonvalidated PTA duration imputations. TBI complications included were elevated intracranial pressure (ICP; none, $<24 \mathrm{~h},>24 \mathrm{~h},>24 \mathrm{~h}$, and sustained, not monitored), whether a craniectomy or craniotomy was performed, and computerized tomography (CT) evidence of focal intracranial injury defined as subdural or epidural hematoma, or intraparenchymal hemorrhage classified as contusion (as opposed to petechial hemorrhage consistent with diffuse axonal injury). Finally, acute care hospital LOS was included as an easily retrievable surrogate marker for associated injury or other comorbidities. 


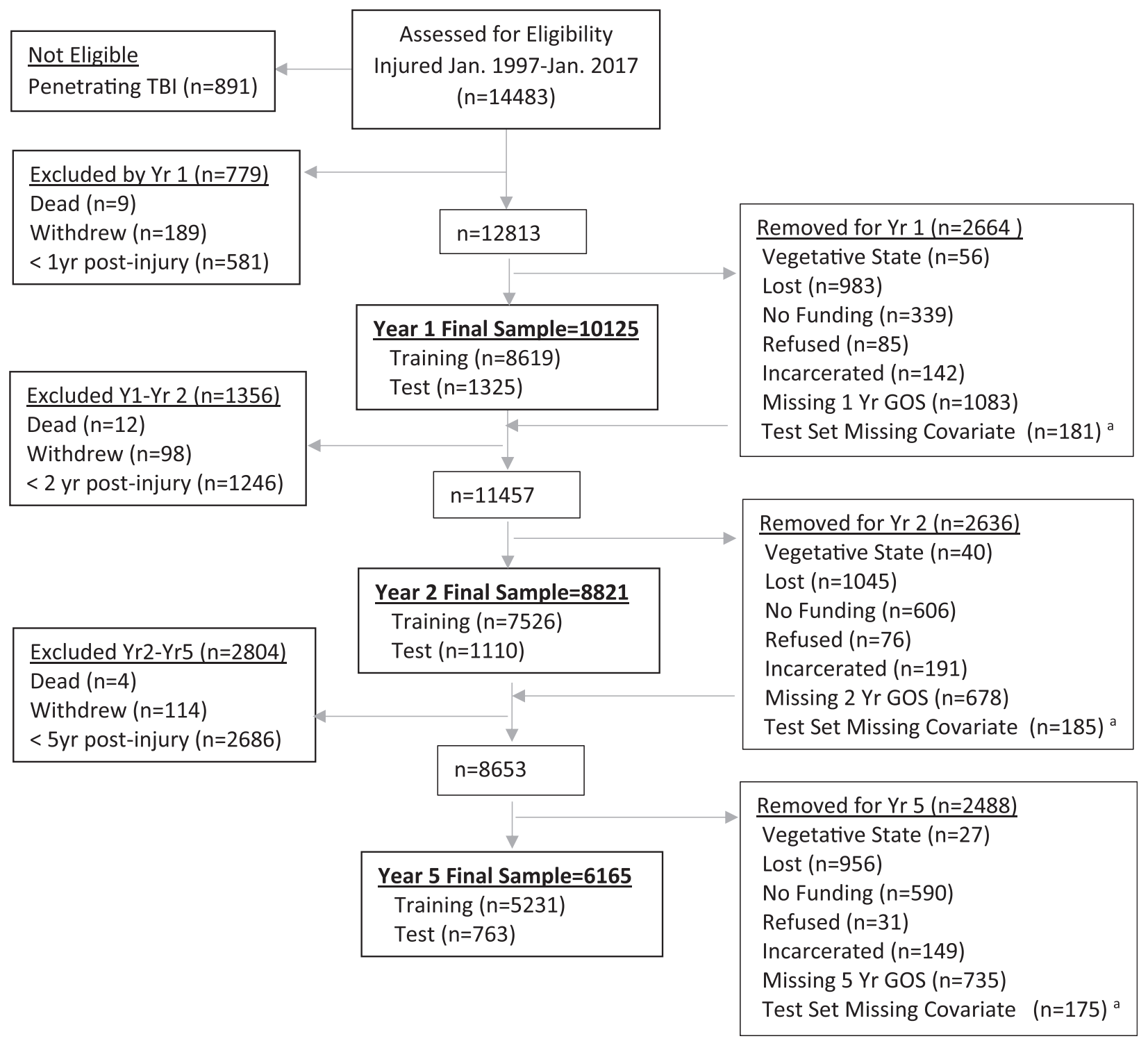

FIG. 1. Participant flow diagram showing samples for the 1-, 2-, and 5-year post-injury models.

${ }^{\text {a }}$ Subjects excluded from the test-set only because of missing values for days in PTA, discharged in PTA, occupational group/ employment, education level, or productivity.

GOS, Glasgow Outcome Scale; LOS, length of stay; PTA, post-traumatic amnesia.

\section{Statistical analysis}

For each follow-up period, the data were randomly split with $85 \%$ placed into a training set and $15 \%$ placed into a test set. We used the training sets to build the predictive models and the test sets to assess the generalizability of each one.

Predictive models were built using classification tree methodology, which are intuitive predictors based on branching logic that can provide flexible predictive qualities. Within this paradigm, a node is defined as a group of homogenous participants and a terminal node is a group of homogenous participants that do not split into subgroups. The algorithm searches for splits to maximize the difference in the outcome, thus all variables captured in the classification tree are considered related to the outcome. Variables appearing higher in the tree are more strongly related to the outcome than variables appearing distally. Predictions can be made using outcome distributions in each node.

A tree was created using the Classification Rule with Unbiased Interaction Selection and Estimation (CRUISE) algorithm sepa- rately at each follow-up time point. ${ }^{27}$ This algorithm allows for splits in two or more directions and accommodates continuous and categorical predictors. Within this algorithm, we used the following options: univariate splits, two-dimensional method, splitting by discriminant analysis, equal priors and equal costs of misclassification, a standard error limit of 0.5 for pruning, and node-wise imputation and fit. To achieve a parsimonious model, nodes containing under 200 observations were not permitted to split.

Two approaches were considered for model building: step-wise restriction of primary branching to PTA duration versus entering all predictor candidates simultaneously. We opted for the lessrestrained modeling because PTA duration was consistently the primary branching in test runs. We also incorporated "manual pruning," the removal of nodes with questionable value along with subsequent nodes, under unanimous consent of a nationally known expert panel.

After the tree was created using the CRUISE algorithm, the percentage of observations in each terminal node were calculated and used to calculate an $M$ statistic and $c$-index to measure the 
Table 1. Description of Glasgow Outcome Scale Levels

\begin{tabular}{ll}
$\begin{array}{l}\text { Glasgow Coma } \\
\text { Scale level }\end{array}$ & \multicolumn{1}{c}{ Brief description } \\
\hline $\begin{array}{l}\text { Dead } \\
\text { Vegetative } \\
\text { state }\end{array}$ & $\begin{array}{l}\text { N/A } \\
\text { Condition of unawareness with only reflex } \\
\text { responses but with periods of spontaneous } \\
\text { eye opening } \\
\text { Severe } \\
\text { disability }\end{array}$ \\
$\begin{array}{l}\text { Dependent for daily support for mental } \\
\text { and/or physical disability } \\
\text { disability }\end{array}$ & $\begin{array}{l}\text { Some disability but able to look after } \\
\text { themselves; independent at home but } \\
\text { dependent outside }\end{array}$ \\
Good recovery & $\begin{array}{l}\text { Resumption of normal life with the capacity } \\
\text { to work even if pre-injury status has not } \\
\text { been achieved; may have minor neurological } \\
\text { or psychological deficits }\end{array}$
\end{tabular}

N/A, not applicable. predictability of each tree. ${ }^{28}$ This statistic is calculated by averaging the c-statistics from each of the ordinal comparisons and ranges from 0.5 (random predictability) to 1.0 (perfect prediction). Confidence intervals (CIs) for the $c$-index were computed using a nonparametric bootstrap based on 500 bootstrap samples. The predictability of each classification tree was then assessed within the independent test set.

\section{Results}

\section{Independent variables}

Table 2 summarizes relevant characteristics of all participants eligible for the year-1 GOS analyses (i.e., lacking any of these exclusions: penetrating TBI, death, withdrew, or under 1-year postinjury), showing overall statistics for the entire sample as well as the divided training and testing sets. Unsurprisingly, the characteristics mirror past publications from the TBIMS. ${ }^{29,30}$ The sample was predominately male $(72.9 \%)$ with a median (IQR) age at injury of $39.0(24.0-55.5)$ years. Although not predictor candidates, $9.9 \%$ were of Hispanic ethnicity and $29.6 \%$ were minority races (nonwhite).

Table 2. Predictor Candidate Variables on Those Eligible at 1 Year Post-Injury $(N=12,813)$

\begin{tabular}{|c|c|c|c|c|}
\hline Characteristic & Level & Overall & Training set & Test set \\
\hline Age at injury & & $39(24-56)$ & $39(24-56)$ & $38(24-55)$ \\
\hline \multirow{2}{*}{ Sex } & Female & $3470(27.1 \%)$ & $2968(27.2 \%)$ & $502(26.2 \%)$ \\
\hline & Male & $9341(72.9 \%)$ & $7930(72.8 \%)$ & $1411(73.8 \%)$ \\
\hline \multirow[t]{2}{*}{ Prior TBI } & Yes & $1797(14.0 \%)$ & $1489(13.7 \%)$ & $308(16.1 \%)$ \\
\hline & No & $11,016(86.0 \%)$ & $9411(86.3 \%)$ & $1605(83.9 \%)$ \\
\hline Number of past TBI & & $0(0-0)$ & $0(0-0)$ & $0(0-0)$ \\
\hline \multirow[t]{3}{*}{ Education } & $<\mathrm{HS} / \mathrm{GED}$ & $2766(23.9 \%)$ & $2381(24.3 \%)$ & $385(22.2 \%)$ \\
\hline & HS/GED & $4122(35.7 \%)$ & $3476(35.4 \%)$ & $646(37.2 \%)$ \\
\hline & $>$ HS/GED & $4670(40.4 \%)$ & $3962(40.4 \%)$ & $708(40.1 \%)$ \\
\hline \multirow[t]{2}{*}{ Productivity } & Yes & $7684(69.3 \%)$ & $6529(69.2 \%)$ & $1155(69.7 \%)$ \\
\hline & No & $3403(30.7 \%)$ & $2900(30.8 \%)$ & $503(30.3 \%)$ \\
\hline \multirow[t]{4}{*}{ Occupational category } & Professional & $1691(13.9 \%)$ & $1454(14.1 \%)$ & $237(13.1 \%)$ \\
\hline & Skilled & $4166(34.3 \%)$ & $3521(34.1 \%)$ & $645(35.6 \%)$ \\
\hline & Manual labor & $1946(16.0 \%)$ & $1666(16.1 \%)$ & $280(15.4 \%)$ \\
\hline & None & $4334(35.7 \%)$ & $3683(35.7 \%)$ & $651(35.9 \%)$ \\
\hline \multirow[t]{2}{*}{ Problem alcohol use } & Yes & $1783(15.3 \%)$ & $1518(15.3 \%)$ & $265(15.3 \%)$ \\
\hline & No & $9899(84.7 \%)$ & $8429(84.7 \%)$ & $1470(84.7 \%)$ \\
\hline \multirow{2}{*}{ Illicit drug use } & Yes & $2446(19.5 \%)$ & $2056(19.3 \%)$ & $390(21.0 \%)$ \\
\hline & No & $10,074(80.5 \%)$ & $8604(80.7 \%)$ & $1470(79.0 \%)$ \\
\hline PTA duration & & $22(9-41)$ & $23(9-41)$ & $21(8-40)$ \\
\hline \multirow[t]{2}{*}{ Discharged in PTA } & Yes & $2343(18.3 \%)$ & $2001(18.4 \%)$ & $342(17.9 \%)$ \\
\hline & No & $10,470(81.7 \%)$ & $8899(81.6 \%)$ & $1571(82.1 \%)$ \\
\hline Initial motor GCS & & $6(4-6)$ & $6(4-6)$ & $6(4-6)$ \\
\hline \multirow{5}{*}{ Elevated ICP } & None & $3384(26.7 \%)$ & $2902(26.9 \%)$ & $482(25.5 \%)$ \\
\hline & $<24 \mathrm{~h}$ & $1175(9.3 \%)$ & $1021(9.5 \%)$ & $154(8.1 \%)$ \\
\hline & $>24 h$ & $1316(10.4 \%)$ & $1134(10.5 \%)$ & $182(9.6 \%)$ \\
\hline & $>24 \mathrm{~h}$ sustained & $290(2.3 \%)$ & $243(2.3 \%)$ & $47(2.5 \%)$ \\
\hline & Not monitored & $6500(51.3 \%)$ & $5474(50.8)$ & $1026(54.3 \%)$ \\
\hline \multirow[t]{2}{*}{ Craniotomy } & Yes & $1544(12.1 \%)$ & $1319(12.1 \%)$ & $225(11.8 \%)$ \\
\hline & No & $11,269(87.9 \%)$ & $9581(87.9 \%)$ & $1688(88.2 \%)$ \\
\hline \multirow[t]{2}{*}{ Craniectomy } & Yes & $1000(7.8 \%)$ & $872(8.0 \%)$ & $128(6.7 \%)$ \\
\hline & No & $11,813(92.2)$ & $10,028(92.0 \%)$ & $1785(93.3 \%)$ \\
\hline \multirow{2}{*}{ CT focal hemorrhage } & Yes & $10,015(80.8)$ & $8529(80.9 \%)$ & $1486(80.0 \%)$ \\
\hline & No & $2387(19.2)$ & $2015(19.1 \%)$ & $372(20.0 \%)$ \\
\hline Acute hospital LOS & & $16(9-26)$ & $17(9-26)$ & $16(9-26)$ \\
\hline
\end{tabular}

Continuous variables shown as median (IQR); categorical variables shown as $N(\%)$.

TBI, traumatic brain injury; PTA, post-traumatic amnesia; GCS, Glasgow Coma Scale; ICP, intracranial pressure; CT, computed tomography; LOS, length of stay; HS, high school; GED, General Educational Development. 
Median PTA duration for the entire sample, including the $18.3 \%$ still in PTA at last hospital observation, was 22.0 days.

\section{Glasgow Outcome Scale category distribution across outcome years}

The distribution of GOS categories improved over time. In the larger training set, SD sequentially declined from $31.8 \%$ at 1-year, to $26.9 \%$ at 2 -year, to $21.8 \%$ at 5 -year post-injury, whereas GR increased from $35.9 \%$, to $39.5 \%$, to $42.0 \%$ (node 0; Figs. 2-4). A cross-year analysis in the test and training sets comparing sample characteristics suggested the GOS change over time was from patients improving rather than biased dropout (see Supplementary Tables S1-S8).

\section{Classification trees developed in training set}

Each preliminary decision tree was inspected for pruning decisions. No pruning was implemented in the year- 1 or year- 5 model, but we pruned off two overly cumbersome later-stage branches that appeared spurious in the year-2 model; neither involved a unique predictor. Even after pruning, the year- 2 model had the greatest branching complexity. The full unpruned year- 2 tree is shown in the supplementary tables (see online supplementary material at http://www.liebertpub.com)

The final prognostic models are displayed in Figures 2-4 for the year-1, -2 , and -5 GOS, respectively, with terminal nodes denoted by solid lined boxes. As the figures show, PTA duration was uniformly the most important splitting variable in our final GOS prediction models and relatively few other candidate variables contributed. Besides PTA duration and the dichotomous discharge PTA status variable, no injury-related variable (initial motor GCS, focal hemorrhage on CT, elevated ICP, cranial surgery, and acute hospital LOS) appeared in any model. For non-injury-related variables, age at injury, pre-morbid education level, occupational category, and productivity each contributed to at least one model, whereas past TBI, pre-morbid problem alcohol use, and illicit drug use did not. Age tended to occupy the upper hierarchal position compared with education, occupation, or productivity. Generally, patients who spent fewer days in PTA, were younger at injury, employed, or productive pre-injury, and had more education pre-injury had better outcomes.

Supplementary Tables 3-8 contain additional details on the terminal nodes of the final classification tree models, including CIs for the GR/MD/SD distribution, and complete eight-category GOSE breakdown (i.e., upper and lower subdivisions of GR, MD, and $\mathrm{SD}$, as well as vegetative state and death). (see online supplementary material at http://www.liebertpub.com)

\section{Prediction model performance}

As measured by the C-statistic, the 2-year GOS classification tree showed highest predictability (training, $\mathrm{c}=0.731 ; 95 \% \mathrm{CI}$, $0.724,0.738$; test, $\mathrm{c}=0.730[0.711,0.749])$. Predictability values for year-1 were training: $c=0.700(0.693,0.707)$ and test: $c=0.691$ $(0.675,0.711)$ and for year-5 were training: $c=0.712(0.702,0.722)$ and test: $c=0.698(0.675,0.720)$. These values indicate reasonable predictability for all the models. ${ }^{31}$

\section{Discussion}

Reinforcing past research, the current study demonstrates the unique potential for late improvement after moderate-to-severe TBI, with GOS distribution improving from year-1 to year-2, as well as the less studied year- 2 to year-5 interval. ${ }^{32}$ This late im- provement suggests that long-term prognostic models need to be time-point specific, which this study sought to build.

Throughout our decision trees, PTA duration consistently dominated branching hierarchy, with longer PTA portending worse GOS prognosis. PTA duration, the interval from injury until return of full orientation or new memory carryover, is easily measured clinically and is a proven TBI severity indicator and outcome predictor. ${ }^{11,13,33}$ Figures 2-4 show PTA's influence quantitatively across years by way of specific location of branching cutpoints and GOS distribution predictions. In addition to being the main predictor, PTA duration was the only injury-related variable influencing GOS prediction. Failing to contribute were initial motor GCS, focal hemorrhagic mass lesion on head CT, elevated ICP, cranial surgery, and acute hospital LOS. The supremacy of PTA duration may stem from these other severity markers being precursors for PTA and/or because PTA duration incorporates more rate of recovery information. ${ }^{33}$ Regardless, our findings corroborate PTA duration as a robust, and single best predictor of long-term functional outcome after moderate-tosevere closed TBI.

Although few secondary predictors contributed to our final trees, their variation between PTA branches indicates that the degree of their influence depends on the severity of TBI as measured by PTA duration. Age was the leading secondary predictor in our trees, generally occupying the upper hierarchical position when multiple secondary predictors appeared. This finding is consistent with existing TBI outcomes research, with age almost always being found an important predictor. ${ }^{17,18}$ Other contributory variables were premorbid education, productivity, and occupation. Some researchers have opined that these socioeconomic variables may indirectly influence outcome by marking innate intelligence, personality or psychological characteristics, brain reserve capacity, and/or social support systems. ${ }^{34,35}$ Neither sex nor pre-morbid alcohol/drug misuse contributed at any year despite some past research showing poorer outcomes in males or substance misusers. ${ }^{17,36}$ Possibly, their influence was over-ridden by the other socioeconomic variables included. Past TBI may not have contributed because it generally captured injuries much milder than the current incident of moderate-to-severe TBI.

In comparing the best prognostic group for each model year (shortest PTA duration branch), the upper cutpoint for PTA increased from 16 days at year-1, to 18 days at year-2, to 20 days at year-5. Despite this elongation of PTA duration, GOS improved sequentially within this branch. At year-2 and year-5, but not year1 , age provided further prognostic separation, with better GOS for younger ages. This delayed influence of age suggests a recovery plateau for older patients relative to younger patients between year1 and year-2 for shorter PTA durations. Taken together, emerging from PTA in under 3 weeks portends a relatively favorable longterm functional prognosis, especially for younger ages with a horizon beyond 1 year. Pre-morbid productivity offers additional predictability at year- 2 and year-5, as does education at year-2. As a cautionary note, the highest GR rate in any prognostic group is $65.7 \%$, indicating the seriousness of moderate-to-severe TBI. Further, achieving GR on GOS does not stipulate full brain recovery, given that persisting cognitive decrements relative to preinjury are ubiquitous after severe TBI. ${ }^{37}$

In the worst prognostic group (longest PTA duration branch), the lower cutpoint for PTA also varied across outcome years, but the pattern did not mirror the best prognostic group upper cutpoint. After increasing from 47 days at year- 1 to 58 days at year-2, the lower cutpoint dropped to $>44$ days at year -5 within this branch. 


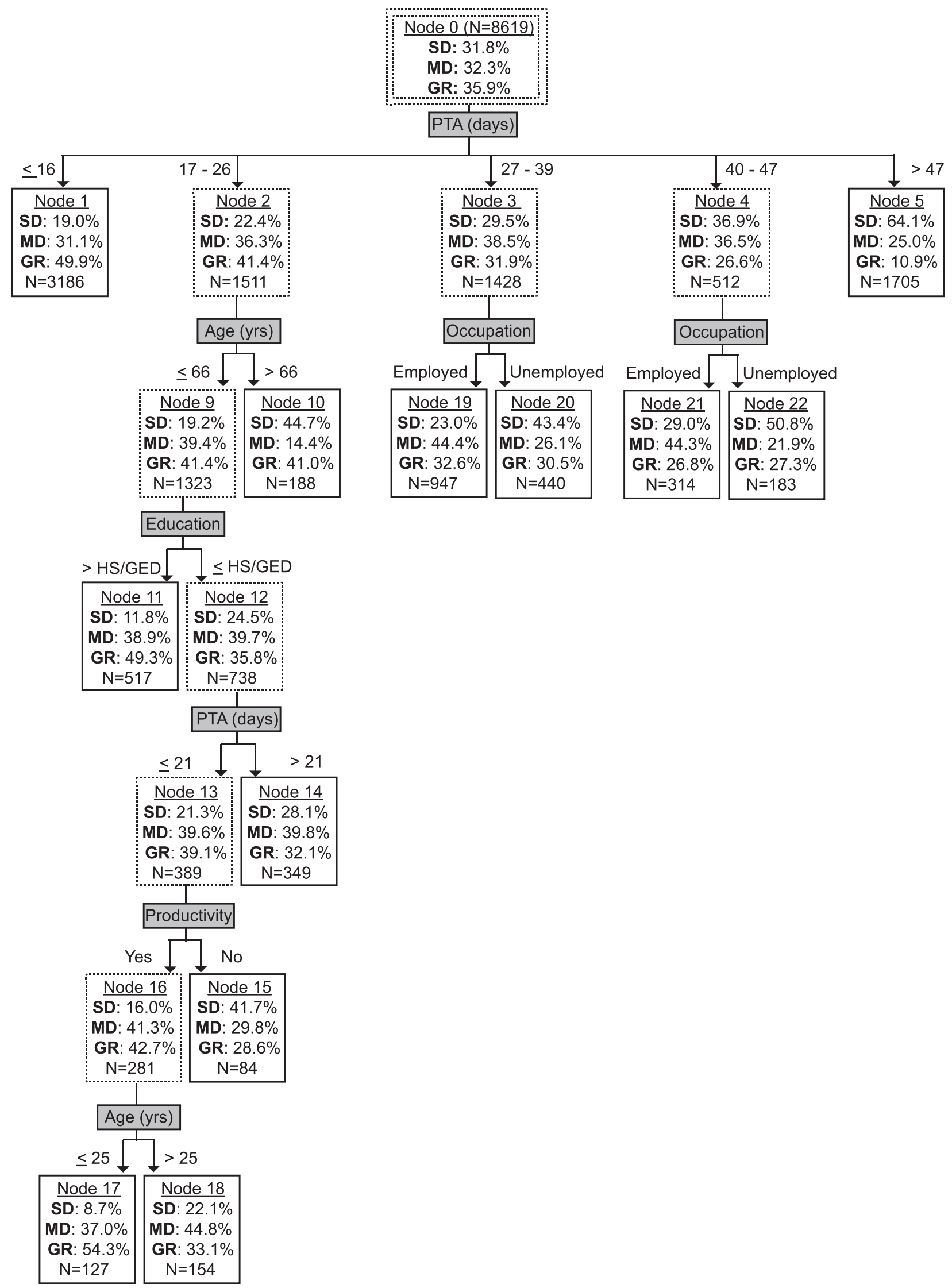

FIG. 2. Prognostic model for 1-year post-injury GOS.

Nodes with solid lines represent terminal nodes. Sample sizes correspond to the training set and may not sum to higher-order nodes due to missing values.

GOS, Glasgow Outcome Scale; PTA, post-traumatic amnesia; SD, severe disability; MD, mild disability; GR, good recovery. 


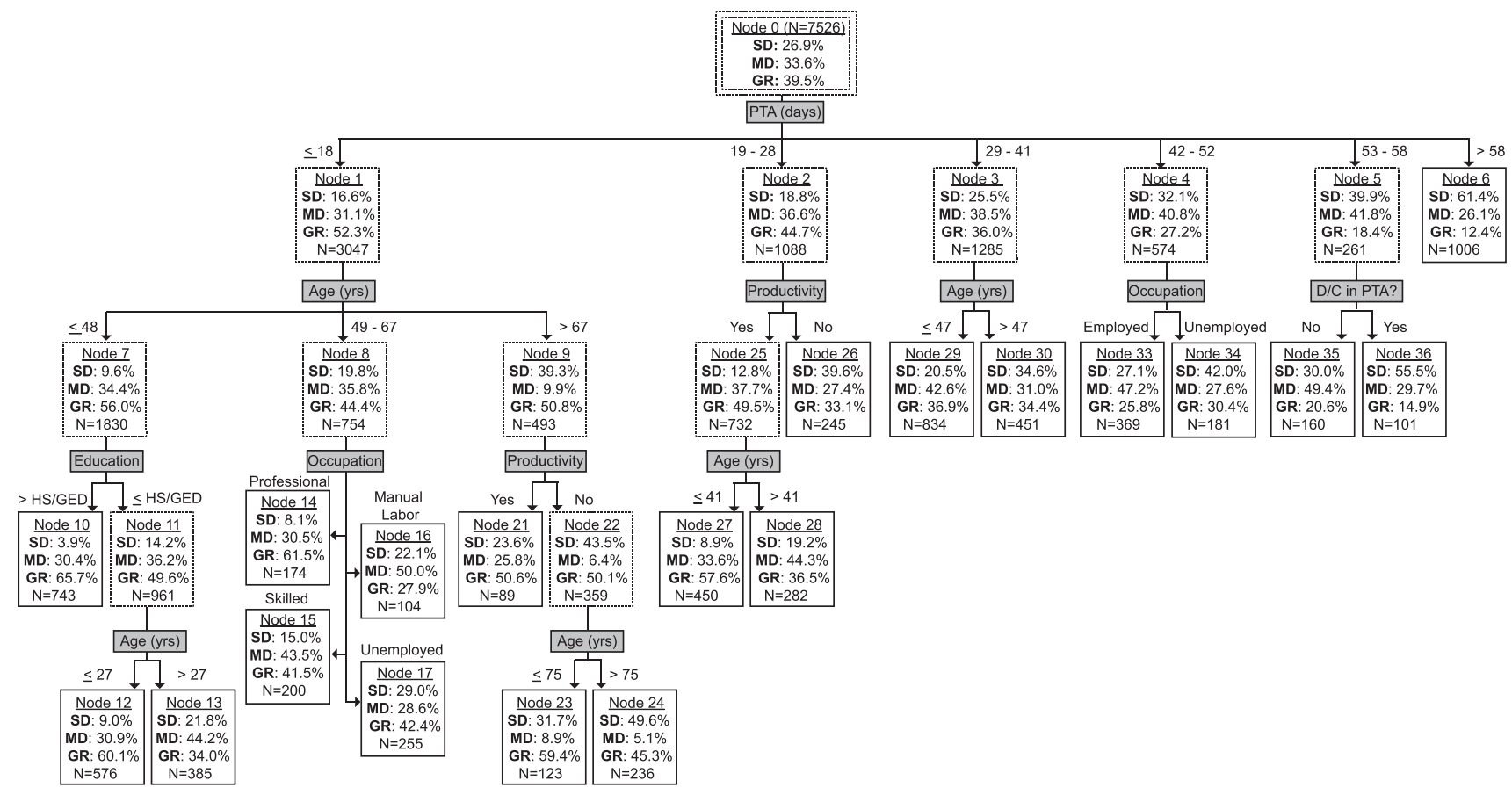

FIG. 3. Prognostic model for 2-year post-injury GOS.

Nodes with solid lines represent terminal nodes. Sample sizes correspond to the training set and may not sum to higher-order nodes because of missing values.

GOS, Glasgow Outcome Scale; PTA, post-traumatic amnesia; SD, severe disability; MD, mild disability; GR, good recovery.

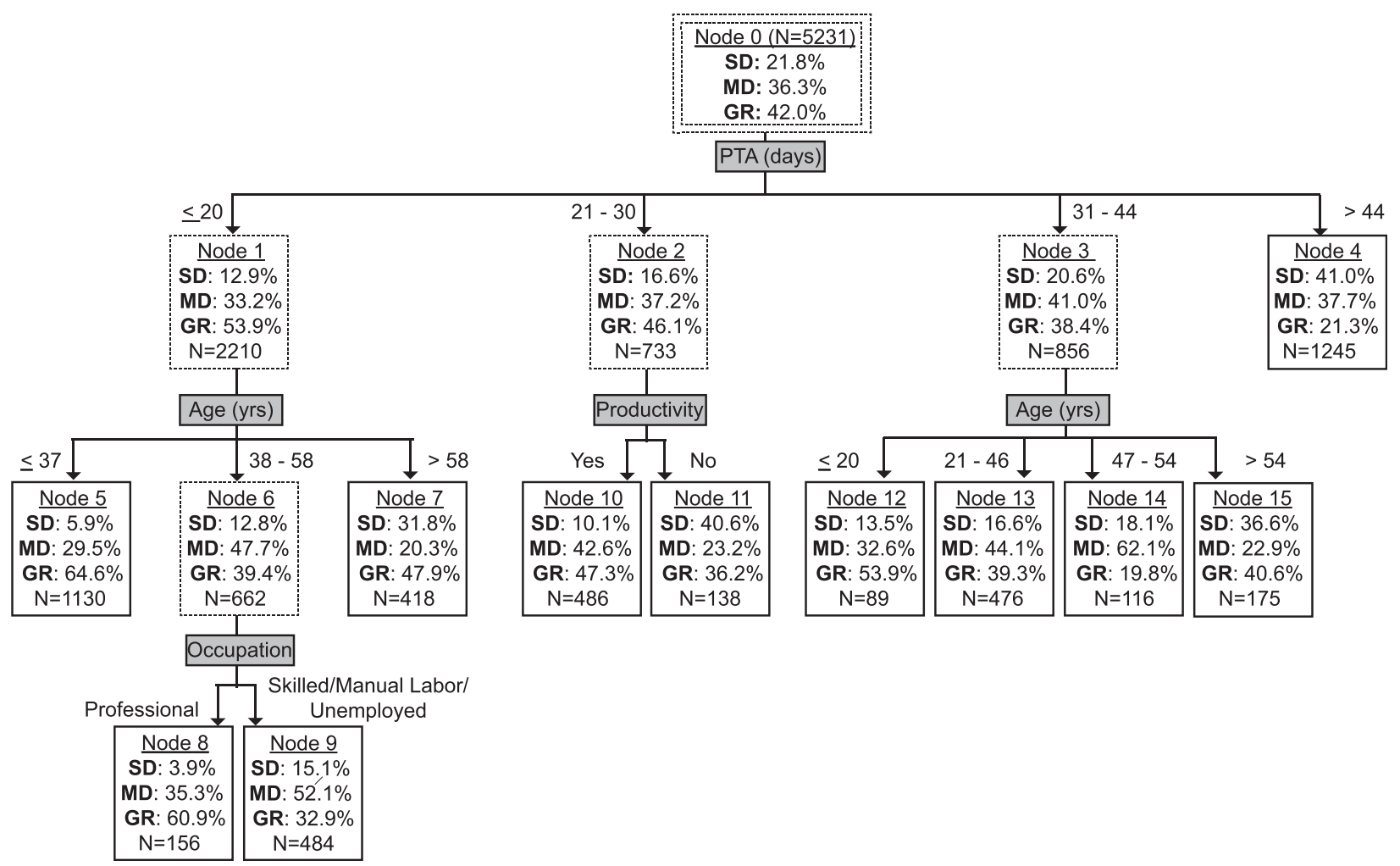

FIG. 4. Prognostic model for 5-year post-injury GOS.

Nodes with solid lines represent terminal nodes. Sample sizes correspond to the training set and may not sum to higher-order nodes because of missing values.

GOS, Glasgow Outcome Scale; PTA, post-traumatic amnesia; SD, severe disability; MD, mild disability; GR, good recovery. 
This drop may be an artifact of truncated still-in-PTA measurements being overweighed in these branches. Supporting this, the binary still-in-PTA variable was the sole secondary predictor for the 52-58 days PTA branch at year-2, implying the purer lower cutpoint here is 52 days or longer. Regardless, group GOS improvement occurred sequentially from year-1, to year-2, to year-5. Interestingly, no secondary branching existed for any year in the longest PTA branches. This finding suggests a PTA duration threshold beyond which the TBI is so severe that it negates any secondary predictor influences, at least within the constraints of our modeling technique and sample sizes. Our data indicate that this threshold is around 7 weeks.

This study's strengths were flexible modeling methods, inherent clinical applicability of findings, large multi-center cohort size, and verification of predictably within a separate data set. The TBIMS NDB participants are enrolled from 16 inpatient rehabilitation facilities (IRFs) geographically dispersed throughout the continental United States and have similar acute care length of stay and similar functional deficits as those admitted to non-TBIMS IRFs in the United States, ${ }^{38,39}$ thus, our findings should generalize to patients from non-TBIMS IRFs. However, several limitations are noteworthy. Like any TBIMS analysis, findings may not extend to patients with TBI not qualifying for inpatient rehabilitation because they either did not have significant self-care deficits (i.e., injury too mild), were unable to participate (i.e., injury overly severe), or were not referred or deemed eligible for other reason(s). Retention rate was high for a comprehensive longitudinal study, but the analysis samples may have been biased by missing data, particularly the lack of GOS interview data for retained participants who chose the mail-in assessment. Relatedly, nonrandom dropout may have impacted findings on the influence of predictor variables, especially substance abuse history given past research showing that substance abusers miss follow-up visits more often than those without substance abuse issues. ${ }^{38}$ Our decision-tree modeling may have limited predictive power compared to regression or other mathematical modeling. ${ }^{8}$ However, ours permitted greater than twoway splitting of nodes, an advancement in decision-tree methods from previous TBI outcomes research. Using the more distinct and easier-tointerpret main GOS categories, ${ }^{40}$ versus the finer-grained GOSE subcategories, may have masked predictive information. Limited statistical power as node sizes diminished may have prevented more secondary branches of potential clinical importance. The $\mathrm{C}$-statistic results were not ideal, underscoring the importance of interpretive flexibility and avoiding absolutism in forecasting functional outcome. Whether additional significant variance could be explained by other clinically available premorbid or injury variables not available in TBIMS database is unknown. Of likely greater influence are downstream factors unknown at baseline, such as post-injury development of depression or changes in alcohol use or social support over time.

Another limitation was the focus on functional outcomes, so our models may not apply to individuals who are dead or vegetative at given follow-up points. We recommend that, when these models are used in practice, an honest discussion be had with the patient and/or caregiver about the incidences of vegetative state or death post-injury, particularly for the groups of patients represented by the nodes with the most SD outcomes. (see online supplementary material at http://www.liebertpub.com)

Last, one injury variable we intentionally excluded was length of coma (LOC), which is measured as time until following motor commands in the TBIMS NDB. Our rationale was that LOC can be more difficult for clinicians to extract from chart review, and it is strongly correlated with PTA duration but offers less predictability. ${ }^{41,42}$ Further, within the TBIMS NDB, the distribution of LOC is highly skewed with major clumping at zero to 1 day, and LOC has more missing observations than PTA duration. Nonetheless, LOC as an alternative to PTA duration offers the potential to provide prognosis at an earlier time post-injury (i.e., when still in PTA). Our models can be applied to some individuals still in PTA, but only upon or after rehabilitation discharge. Thus, we plan future study of our classification-tree modeling that replaces PTA duration with LOC.

\section{Conclusion}

This study developed decision trees for gaining prognostic information on meaningful long-term functional outcomes after moderate-severe closed TBI and demonstrated reasonable predictability in a separate test sample. There are many potential benefits of this easy-to-use tool: helping providers set expectations and plan rehabilitation treatments; patient selection and stratification in future clinical trials; assisting patients and families with resource planning; and addressing their emotional burden of future uncertainty. Future research is needed to assess external validity in separate cohorts.

\section{Acknowledgments}

This study was supported by National Institutes of Health (NIH) \#RHD089097A.

The data contents for this manuscript were developed under a grant from the National Institute on Disability, Independent Living, and Rehabilitation Research (NIDILRR; grant number 90 DP0013). NIDILRR is a Center within the Administration for Community Living (ACL), Department of Health and Human Services (HHS). Post-doctoral fellows were supported by NIDILRR grant number 90AR5025.

The contents of this manuscript do not necessarily represent the policy of NIH or NIDILRR, ACL, HHS, and you should not assume endorsement by the Federal Government.

Dr. William C. Walker had full access to all the data in the study and takes responsibility for the integrity of the data and the accuracy of the data analysis.

\section{Author Disclosure Statement}

No competing financial interests exist.

\section{Supplementary Material}

Supplementary Table S1

Supplementary Table S2

Supplementary Table S3

Supplementary Table S4

Supplementary Table S5

Supplementary Table S6

Supplementary Table S7

Supplementary Table S8

\section{References}

1. Beauchamp, K., Mutlak, H., Smith, W.R., Shohami, E., and Stahel, P.F. (2008). Pharmacology of traumatic brain injury: where is the "golden bullet"? Mol. Med. 14, 731-740.

2. Menon, D.K. (2009). Unique challenges in clinical trials in traumatic brain injury. Crit. Care. Med. 37, 1 Suppl., S129-S135.

3. Lefebvre, H., and Levert, M.J. (2012). The close relatives of people who have had a traumatic brain injury and their special needs. Brain Inj. 26, 1084-1097.

4. Bonds, B.W., Dhanda, A., Wade, C., Massetti, J., Diaz, C., and Stein, D.M. (2015). Prognostication of mortality and long term functional outcomes following traumatic brain injury: can we do better? J. Neurotrauma. Jul 31. doi: 10.1089/neu.2014.3742. [Epub ahead of print]

5. Jennett, B., and Bond, M. (1975). Assessment of outcome after severe brain damage. Lancet $1,480-484$. 
6. Wilson, J.T., Pettigrew, L.E., and Teasdale, G.M. (1998). Structured interviews for the Glasgow Outcome Scale and the Extended Glasgow Outcome Scale: guidelines for their use. J. Neurotrauma 15, 573-585.

7. Mushkudiani, N.A., Hukkelhoven, C.W., Hernandez, A.V., Murray, G.D., Choi, S.C., Maas, A.I., and Steyerberg, E.W. (2008). A systematic review finds methodological improvements necessary for prognostic models in determining traumatic brain injury outcomes. J. Clin. Epidemiol. 61, 331-343.

8. Hukkelhoven, C.W., Rampen, A.J., Maas, A.I., Farace, E., Habbema, J.D., Marmarou, A., Marshall, L.F., Murray, G.D., and Steyerberg, E.W. (2006). Some prognostic models for traumatic brain injury were not valid. J. Clin. Epidemiol. 59, 132-143.

9. Andrews, P.J., Sleeman, D.H., Statham, P.F., McQuatt, A., Corruble, V., Jones, P.A., Howells, T.P., and Macmillan, C.S. (2002). Predicting recovery in patients suffering from traumatic brain injury by using admission variables and physiological data: a comparison between decision tree analysis and logistic regression. J. Neurosurg. 97, 326-336.

10. Choi, S.C., Muizelaar, J.P., Barnes, T.Y., Marmarou, A., Brooks, D.M., and Young, H.F. (1991). Prediction tree for severely headinjured patients. J. Neurosurg. 75, 251-255.

11. Brown, A.W., Malec, J.F., McClelland, R.L., Diehl, N.N., Englander, J., and Cifu, D.X. (2005). Clinical elements that predict outcome after traumatic brain injury: a prospective multicenter recursive partitioning (decision-tree) analysis. J. Neurotrauma 22, 1040-1051.

12. Harrison-Felix, C., Newton, C.N., Hall, K.M., and Kreutzer, J.S. (1996). Descriptive findings from the traumatic brain injury model systems national data base. J. Head Trauma Rehabil. 11, 1-14.

13. Sherer, M., Struchen, M.A., Yablon, S.A., Wang, Y., and Nick, T.G. (2008). Comparison of indices of traumatic brain injury severity: Glasgow Coma Scale, length of coma and post-traumatic amnesia. J. Neurol. Neurosurg. Psychiatry 79, 678-685.

14. Walker, W.C., Ketchum, J.S. III, Marwitz, J.H., Kolakowsky-Hayner, S.A., McClish, D.K., and Bushnik, T. (2015). Global outcome and late seizures after penetrating versus closed traumatic brain injury: a NIDRR TBI model systems study. J. Head Trauma Rehabil. 30, 231-240.

15. Wilson, J.T. (2001). Assessing outcome in head injury trials. Curr. Pharm. Des. 7, 1537-1552.

16. Kothari, S. (2006). Prognosis after severe TBI: a practical, evidence-based approach, in: Brain Injury Medicine: Principles and Practice. R. Zafonte, N.D. Zasler, and D.I. Katz (eds). Demos: New York, pps. 529-544.

17. Cuthbert, J.P., Pretz, C.R., Bushnik, T., Fraser, R.T., Hart, T., Kolakowsky-Hayner, S.A., Malec, J.F., O’Neil-Pirozzi, T.M., and Sherer, M. (2015). Ten-year employment patterns of working age individuals after moderate to severe traumatic brain injury: a National Institute on Disability and Rehabilitation Research Traumatic Brain Injury Model Systems study. Arch. Phys. Med. Rehabil. 96, 2128-2136.

18. Corrigan, J.D., Cuthbert, J.P., Harrison-Felix, C., Whiteneck, G.G., Bell, J.M., Miller, A.C., Coronado, V.G., and Pretz, C.R. (2014). US population estimates of health and social outcomes 5 years after rehabilitation for traumatic brain injury. J. Head Trauma Rehabil. 29, E1-E9.

19. Cuthbert, J.P., Harrison-Felix, C., Corrigan, J.D., Bell, J.M., HaarbauerKrupa, J.K., and Miller, A.C. (2015). Unemployment in the United States after traumatic brain injury for working-age individuals: prevalence and associated factors 2 years postinjury. J. Head Trauma Rehabil. 30, 160-174.

20. Sherer, M., Nick, T.G., Sander, A.M., Hart, T., Hanks, R., Rosenthal, M., High, W.M., Jr., and Yablon, S.A. (2003). Race and productivity outcome after traumatic brain injury: influence of confounding factors. J. Head Trauma Rehabil. 18, 408-424.

21. Corrigan, J., and Bogner, J. (2007). Initial reliability and validity of the Ohio State University TBI identification method. J. Head Trauma Rehabil. 22, 318-329.

22. Bogner, J., and Corrigan, J. (2009). Reliability and predictive validity of the Ohio State University TBI identification method with prisoners J. Head Trauma Rehabil. 24, 279-291.

23. Walker, W.C., Marwitz, J.H., Kreutzer, J.S., Hart, T., and Novack, T. (2006). Occupational categories and return to work after traumatic brain injury: a multi-center study. Arch. Phys. Med. Rehabil. 87, 1576-1582.

24. Centers for Disease Control and Prevention. (1988). Behavioral Risk Factor Surveillance System user's guide. Centers for Disease Control and Prevention: Atlanta, GA.

25. Cahalan, D., and Cisin, I.H. (1968). American drinking practices: summary of findings from a national probability sample. II. Measurement of massed versus spaced drinking. Q. J. Stud. Alcohol 29, 642-656.

26. Healey, C., Osler, T.M., Rogers, F.B., Healey, M.A., Glance, L.G., Kilgo, P.D., Shackford, S.R., and Meredith, J.W. (2003). Improving the Glasgow Coma Scale score: motor score alone is a better predictor. J. Trauma 54, 671-678; discussion, 678-680.

27. Kim, H., and Loh, W.Y. (2001). Classification trees with unbiased multiway splits. J. Am. Stat. Assoc. 96, 589-604.

28. Hand, D., and Till, R. (2001). A simple generalisation of the area under the ROC curve for multiple class classification problems. Mach. Learn. 45, 171-186.

29. Kreutzer, J.S., Marwitz, J.H., Sima, A.P., Bergquist, T.F., JohnsonGreene, D., Felix, E.R., Whiteneck, G.G., and Dreer, L.E. (2016) Resilience following traumatic brain injury: a traumatic brain injury model systems study. Arch. Phys. Med. Rehabil. 97, 708-713.

30. Dams-O'Connor, K., Pretz, C., Billah, T., Hammond, F.M., and Harrison-Felix, C. (2015). Global outcome trajectories after TBI among survivors and nonsurvivors: a National Institute on Disability and Rehabilitation Research Traumatic Brain Injury Model Systems study. J. Head Trauma Rehabil. 30, E1-E10.

31. Hosmer, D.W., and Lemeshow, S. (eds.). (2000). Applied Logistic Regression, 2nd ed. John Wiley \& Sons: New York.

32. Pretz, C.R., and Dams-O'Connor, K. (2013). Longitudinal description of the Glasgow Outcome Scale-Extended for individuals in the Traumatic Brain Injury Model Systems National Database: a National Institute on Disability and Rehabilitation Research Traumatic Brain Injury Model Systems study. Arch. Phys. Med. Rehabil. 94, 2486-2493.

33. Walker, W.C., Ketchum, J.M., Marwitz, J.H., Chen, T., Hammond, F., Sherer, M., and Meythaler, J. (2017). A multicentre study on the clinical utility of post-traumatic amnesia duration in predicting global outcome after moderate-severe traumatic brain injury. J. Neurol. Neurosurg. Psychiatry $81,87-89$

34. Forslund, M.V., Roe, C., Perrin, P.B., Sigurdardottir, S., Lu, J., Berntsen, S., and Andelic, N. (2017). The trajectories of overall disability in the first 5 years after moderate and severe traumatic brain injury. Brain Inj. 31, 329-335.

35. Levi, Y., Rassovsky, Y., Agranov, E., Sela-Kaufman, M., and Vakil, E. (2013). Cognitive reserve components as expressed in traumatic brain injury. J. Int. Neuropsychol. Soc. 19, 664-671.

36. West, S.L. (2011). Substance use among persons with traumatic brain injury: a review. NeuroRehabilitation 29, 1-8.

37. Corrigan, J.D., Horn, S.D., Barrett, R.S., Smout, R.J., Bogner, J., Hammond, F.M., Brandstater, M.E., and Majercik, S. (2015). Effects of patient preinjury and injury characteristics on acute rehabilitation outcomes for traumatic brain injury. Arch. Phys. Med. Rehabil. 96, 8 Suppl., S209-S221.e6.

38. Corrigan, J.D., Harrison-Felix, C., Bogner, J., Dijkers, M., Terrill, M.S., and Whiteneck, G. (2003). Systematic bias in traumatic brain injury outcome studies because of loss to follow-up. Arch. Phys. Med. Rehabil. 84, 153-160.

39. Corrigan, J.D., Cuthbert, J.P., Whiteneck, G.G., Dijkers, M.P., Coronado, V., Heinemann, A.W., Harrison-Felix, C., and Graham, J.E. (2012). Representativeness of the Traumatic Brain Injury Model Systems national database. J. Head Trauma Rehabil. 27, 391-403.

40. Cuthbert, J.P., Corrigan, J.D., Whiteneck, G.G., Harrison-Felix, C., Graham, J.E., Bell, J.M., and Coronado, V.G. (2012). Extension of the representativeness of the Traumatic Brain Injury Model Systems national database: 2001 to 2010. J. Head Trauma Rehabil. 27, E15-E27.

41. Maas, A.I., Braakman, R., Schouten, H.J., Minderhoud, J.M., and van Zomeren, A.H. (1983). Agreement between physicians on assessment of outcome following severe head injury. J. Neurosurg. 58, 321-325.

42. Katz, D.I., and Alexander, M.P. (1994). Traumatic brain injury. Predicting course of recovery and outcome for patients admitted to rehabilitation. Arch. Neurol. 51, 661-670.

43. Sherer, M., Struchen, M.A., Yablon, S.A., Wang, Y., and Nick, T.G. (2008). Comparison of indices of traumatic brain injury severity: Glasgow Coma Scale, length of coma and post-traumatic amnesia. J. Neurol. Neurosurg. Psychiatry, 6, 678-685.

Address correspondence to: William C. Walker, MD Department of Physical Medicine and Rehabilitation Virginia Commonwealth University Box 980677 Richmond, VA 23298-0677

E-mail: william.walker@vcuhealth.org 